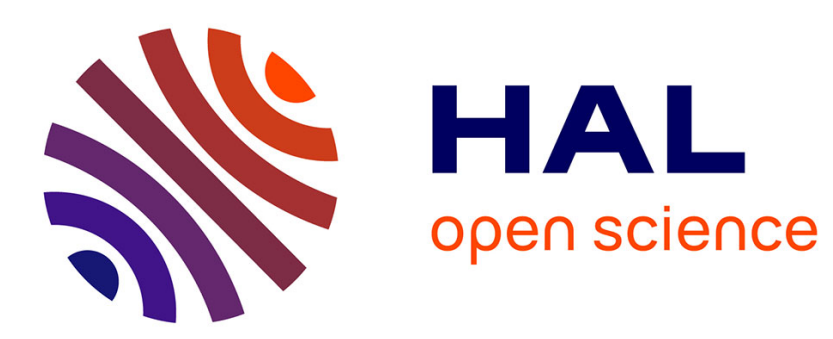

\title{
Eye safe large field of view homodyne detection using a photorefractive CdTe:V crystal
}

Louis-Anne de Montmorillon, Ivan Biaggio, Philippe Delaye, Jean-Claude Launay, Gérald Roosen

\section{To cite this version:}

Louis-Anne de Montmorillon, Ivan Biaggio, Philippe Delaye, Jean-Claude Launay, Gérald Roosen. Eye safe large field of view homodyne detection using a photorefractive CdTe:V crystal. Optics Communications, 1996, 129, pp.293-300. 10.1016/0030-4018(96)00190-3 . hal-00677555v2

\section{HAL Id: hal-00677555 \\ https://hal-iogs.archives-ouvertes.fr/hal-00677555v2}

Submitted on 30 Mar 2012

HAL is a multi-disciplinary open access archive for the deposit and dissemination of scientific research documents, whether they are published or not. The documents may come from teaching and research institutions in France or abroad, or from public or private research centers.
L'archive ouverte pluridisciplinaire HAL, est destinée au dépôt et à la diffusion de documents scientifiques de niveau recherche, publiés ou non, émanant des établissements d'enseignement et de recherche français ou étrangers, des laboratoires publics ou privés. 


\title{
EYE SAFE LARGE FIELD OF VIEW HOMODYNE DETECTION USING A PHOTOREFRACTIVE CdTe:V CRYSTAL
}

\author{
Louis-Anne de Montmorillon, Ivan Biaggio, Philippe Delaye, \\ Jean-Claude Launay ${ }^{*}$, Gérald Roosen
}

Institut d'Optique, Unité de Recherche Associée 14 au Centre National de la Recherche Scientifique, Bât.503, Centre Scientifique d'Orsay, B.P. 147, 91403 Orsay Cedex, France, Email gerald.roosen@iota.u-psud.fr.

*3-AR-Action Aquitaine de Recherche en Apesanteur, B.P. 11, 33165 Saint Médard en Jalles Cedex, France and ICMCB- Institut de Chimie de la Matière Condensée de Bordeaux, Av du docteur A. Schweitzer, 33608 Pessac Cedex, France.

PACS: A8170C, A0760L, A4240K, B4340

Keywords: Photorefractive effect, Homodyne detection, Non destructive testing.

\begin{abstract}
We demonstrate a self-adapting homodyne detection system working at a wavelength of $1.55 \mu \mathrm{m}$. The system uses a photorefractive CdTe:V crystal as the element that combines local oscillator and signal beams. The device spontaneously adapts to slow phase and direction changes in the incoming light. Using a pump beam illumination of $66 \mathrm{~mW} \cdot \mathrm{cm}^{-2}$ and a signal power of $25 \mu \mathrm{W}$, we detect phase modulations corresponding to an optical path variation of a few tenths of nanometers at modulation frequencies higher than a cut-off frequency of $15 \mathrm{~Hz}$. The performance of the system is not affected when the signal light consists of scattered light with an "étendue" of up to $0.1 \mathrm{~mm}^{2} \cdot \mathrm{sr}$, limited only by the lenses we are currently using. We obtain a detection limit of $(6.2 \pm 0.4) \times 10^{-7} \mathrm{~nm} \cdot \sqrt{\mathrm{W} / \mathrm{Hz}}$. This is only about 20 times above the theoretical limit of an ideal interferometric system. We show how to scale these results to the optimized crystals and higher laser intensities necessary to obtain higher sensitivity $\left(<10^{-7} \mathrm{~nm} \cdot \sqrt{\mathrm{W} / \mathrm{Hz}}\right)$ and higher cut-off frequencies $(>1 \mathrm{kHz})$.
\end{abstract}




\section{Introduction}

Real time holographic materials are ideally suited for use as adaptive beam-splitters to coherently superpose a phase-modulated signal beam and a local oscillator beam in a homodyne detection scheme. Photorefractive crystals are among the most sensitive materials for real-time holography and were among the first to be proposed for this kind of application [1].

Detection of phase modulated signals using two wave mixing in a photorefractive crystal is possible whenever the amplitude of the phase modulation is much lower than $\pi / 2$ and its frequency is higher than a cut-off frequency characterizing the response time of the photorefractive effect. The crystal acts as the beam-combiner in a classical interferometric system : the diffracted part of the pump beam interferes with the signal beam.

The main advantage of such a device is the adaptativity of the photorefractive effect. The system adapts to distorted wavefronts and to low frequency mechanical disturbances. It allows the use of speckled beams without loss of signal power, thus decidedly improving the limit given by the antenna's theorem [2]. A homodyne detector based on a photorefractive beam-combiner is a very interesting candidate for quality testing of production line parts in an unfriendly industrial environment. The system would detect the ultrasound waves induced by strong laser pulses in mechanical parts, while at the same time adapting to slowly changing environmental conditions. Ultrasound waves arriving on the object to be tested create displacements that are measured by detecting the phase variations of a laser beam reflected by the rough surface of this object. We will show that photorefractive techniques potentially give a better detection limit and a wider phase modulation bandwidth than the best device presently used, i.e. the confocal Fabry-Perot interferometer [3].

Detection of phase [4] and amplitude [5] changes using two wave mixing has already been demonstrated using insulating $[6,7,8]$ and semi-insulating $[9,10]$ photorefractive crystals. This technique has been used for detection of ultrasonic signals $[8,10]$ as well as for the stabilization of holographic recording [11]. In this work we investigate the use of Vanadium doped CdTe crystals to build a self-adaptive homodyne detector working at the eye-safe wavelength of $1.55 \mu \mathrm{m}$ [12]. We also present a comparison between the theoretical [13] and experimental detection limit and frequency response of the system. 
CdTe presents the advantages of a high $\mathrm{n}^{3} \mathrm{r}_{41}$ value of $\approx 113 \mathrm{pm} \cdot \mathrm{V}^{-1}$ [14], a sensitivity in the 1-1.5 $\mu \mathrm{m}$ wavelength range [15] and a low grating formation fluence of $\approx 100 \mu \mathrm{J} \cdot \mathrm{cm}^{-2}[16]$.

\section{Theoretical background}

We consider a two beam coupling experiment with a strong pump beam and a weak signal beam of parallel polarizations. The signal beam is phase modulated, $E_{S}(x=0, t)=E_{S}(0,0) e^{i \varphi(t)}$. We denote with $\mathrm{E}_{\mathrm{s}}$ the complex amplitude of the signal beam. We assume a phase modulation faster than the response time of the photorefractive effect and with a modulation amplitude much smaller than $\pi / 2$. In this limit, the grating written in the crystal corresponds to the one that would be recorded without any phase modulation. In the non depleted pump approximation, the amplitude of the diffracted part of the pump beam only depends on this grating and is $E_{d}(x, t)=\left(e^{\gamma x}-1\right) E_{s}(x, 0)$, where $\gamma=\gamma^{\prime}+i \gamma^{\prime \prime}$ is the amplitude photorefractive gain [13] and $\mathrm{x}$ is the crystal thickness. The amplitude of the detected signal is then given by the sum of the signal beam and of the diffracted portion of the pump beam. The detected intensity is :

$$
\begin{aligned}
I_{s}(x, t) & =\left|e^{i \varphi(t)}+\left(e^{\gamma x}-1\right)\right|^{2} e^{-\alpha x} I_{s}(0,0) \\
& =\left[e^{2 \gamma^{\prime} x}+2\left(e^{\gamma^{\prime} x} \cos \left(\gamma^{\prime \prime} x\right)-1\right)(\cos \varphi(t)-1)+2 e^{\gamma^{\prime} x} \sin \left(\gamma^{\prime \prime} x\right) \sin \varphi(t)\right] e^{-\alpha x} I_{s}(0,0),
\end{aligned}
$$

where $\alpha$ is the crystal absorption.

This shows that the detected signal will be linearly proportional to $\varphi(t)$ as soon as $\gamma^{\prime \prime} \neq 0$. In this case one has $\left|2\left(\mathrm{e}^{\gamma^{\prime} \mathrm{x}} \cos \left(\gamma^{\prime \prime} \mathrm{x}\right)-1\right)(\cos \varphi(\mathrm{t})-1)\right|<<\left|2 \mathrm{e}^{\gamma^{\prime} \mathrm{x}} \sin \left(\gamma^{\prime \prime} \mathrm{x}\right) \sin \varphi(\mathrm{t})\right|$ (as long as $\left.\varphi<<\pi / 2\right)$ and the portion of the signal which is linearly proportional to the phase change becomes dominant, even if the real part $\gamma^{\prime}$ remains appreciable.

To obtain $\gamma^{\prime \prime} \neq 0$ we apply a DC electric field to the crystal. The signal intensity is then given by :

$$
I_{s}(x, t)=e^{-\alpha x} I_{s}(0,0)\left[e^{2 \gamma^{\prime} x}+2 e^{\gamma^{\prime} x} \sin \left(\gamma^{\prime \prime} x\right) \times \varphi(t)\right]
$$

We use a sinusoidal phase modulation $\varphi(t)=\sqrt{2} \cdot \Delta \varphi \cdot \cos (2 \pi f \mathrm{t})$. One obtains the signal to noise ratio (SNR) by dividing the root mean square of the signal and the noise, respectively. In the case where photon noise is the limiting noise source we have : 


$$
\mathrm{SNR}=\frac{\text { signal variation }}{\text { photon noise }}=\sqrt{\frac{2 \eta I_{s}(0,0)}{h v \Delta f}} e^{-\alpha x / 2} \sin \left(\gamma^{\prime \prime} x\right) \frac{4 \pi \delta}{\lambda},
$$

where $\eta$ is the quantum efficiency of the detection scheme and $\Delta \mathrm{f}$ is its bandwidth. $\delta=\frac{\lambda}{4 \pi} \Delta \varphi$ is the root mean square displacement corresponding to the root mean square phase modulation amplitude $\Delta \varphi . \lambda$ is the wavelength of the light, $v=\mathrm{c} / \lambda$ is the light frequency and $\mathrm{h}$ is Planck's constant.

In order to be able to compare the performances of different systems, we introduce a normalized detection limit, $\delta_{\text {lim }}$, expressed in $\mathrm{nm} \cdot \sqrt{\mathrm{W} / \mathrm{Hz}}$. It corresponds to the minimum detectable displacement using a $1 \mathrm{~W}$ power incident signal beam and a $1 \mathrm{~Hz}$ detector bandwidth :

$$
\delta_{\lim }=\delta(S N R=1) \sqrt{\frac{I_{s}(0,0)}{\Delta f}}=\frac{\lambda}{4 \pi} \sqrt{\frac{h v}{2 \eta}} \frac{e^{+\alpha x / 2}}{\sin \left(\gamma^{\prime \prime} x\right)} .
$$

For plane waves, we find that the normalized detection limit in a classical homodyne interferometer is $\delta_{\lim }^{\text {classic }}=\frac{\lambda}{4 \pi} \sqrt{\frac{h v}{2 \eta}}$. At $\lambda=1.55 \mu \mathrm{m}$ and for $\eta=0.76$, which corresponds to the quantum efficiency of our InGaAs detector, one obtains $\delta_{\lim }^{\text {classic }}=3.6 \times 10^{-8} \mathrm{~nm} \cdot \sqrt{\mathrm{W} / \mathrm{Hz}}$.

With a photorefractive beam-combiner, we obtain the same detection limit in the ideal case where $\alpha=0$ and $\gamma^{\prime \prime} \mathrm{x}=\pi / 2$.

Fig. 1 shows the normalized detection limit. We observe that to obtain a detection limit lower than $10^{-7} \mathrm{~nm} \cdot \sqrt{\mathrm{W} / \mathrm{Hz}}$ at the $1.55 \mu \mathrm{m}$ wavelength, the $\alpha / \gamma^{\prime \prime}$ ratio must be less than 2 .

\section{Experimental realization}

A scheme of the experimental set-up is shown in Fig. 2. The displacement of the vibrating material is simulated with an electro-optic phase modulator (Pockels cell). The scattering of the material is simulated with a diffuser used in transmission. A first optical system images the surface of the diffuser onto the photorefractive crystal and a second system of lenses images the plane of the crystal on the detector. Some experiments were made without diffuser. The grating spacing obtained for a collimated signal beam traveling along the optical axis of the system is $\Lambda=3.5 \mu \mathrm{m}$. With the diffuser, we obtain a range of grating spacings from $2.8 \mu \mathrm{m}$ to $4.8 \mu \mathrm{m}$. We use a distributed feed-back laser diode emitting $10 \mathrm{~mW}$ of infrared light at a wavelength $\lambda=1.55 \mu \mathrm{m}$. 
We use two photorefractive CdTe:V samples, B23 and B25. Their characteristics parameters are presented in Table 1. Sample B23 was characterized in a previous work [16]. The electro-optic coefficient of CdTe is $\mathrm{r}_{41}=5.5 \mathrm{pm} \cdot \mathrm{V}^{-1}[14,15]$. Sample B23 is oriented in such a way that the effective electro-optic coefficient is $r_{\text {eff }}=\sqrt{2 / 3} r_{41}$. Clamped (strain-free) and unclamped (stress-free) [17] electro-optic coefficients differ less than by $2 \%[18,19]$ in CdTe and the piezoelectric contribution $[20,21,22]$ can be safely neglected.

We first studied the effectiveness of a DC applied field with the B23 sample. We measured the intensity modulation of the signal without using any diffuser in the path of the signal beam. The signal beam was phase modulated at $1 \mathrm{kHz}$. Due to a residual intensity modulation generated by the Pockels cell, the detected signal is only clearly measurable for an applied voltage above $0.4 \mathrm{kV}$ (applied electric field $\approx 1 \mathrm{kV} \cdot \mathrm{cm}^{-1}$ ). Above this value the signal varies linearly with the applied electric field (Fig. 3, insert).

We applied a voltage of $3.5 \mathrm{kV}$, to obtain $\mathrm{V} / \mathrm{d}=8 \mathrm{kV} \cdot \mathrm{cm}^{-1}$, for the measurement of the detection limit of our device (sample B23). We note d the length of the crystal along the direction of the applied field, corresponding to the electrode separation. We used an InGaAs detector with a quantum efficiency $\eta=0.76$. The detection bandwidth given by the electronics was $\Delta \mathrm{f}=1.33 \mathrm{MHz}$. The incident signal illumination was $25 \mu \mathrm{W}$. We verified that the intensity modulation amplitude of the detected signal was proportional to the phase modulation amplitude given by the electro-optic modulator. For an equivalent root mean square displacement $\delta=2.1 \mathrm{~nm}$ we measured a signal to noise ratio of 16 . From this we extrapolate to a displacement of $0.13 \mathrm{~nm}$ for a signal to noise ratio of 1 and we obtain a detection limit of $5.8 \times 10^{-7} \mathrm{~nm} \cdot \sqrt{\mathrm{W} / \mathrm{Hz}}$. From Eq. (4) we then deduce $\gamma^{\prime \prime}=0.41 \mathrm{~cm}^{-1}$.

From the experimental data one can also obtain the imaginary part of the amplitude photorefractive gain $\gamma^{\prime \prime}$ in a different way by measuring the ratio between the signal modulation amplitude and its DC part. From Eq. (2), this ratio theoretically equals $\frac{8 \pi \delta}{\lambda} \frac{\sin \gamma^{\prime \prime} x}{e^{\gamma^{\prime} x}}$. From classical energy transfer measurement by two wave mixing, we deduce $\gamma^{\prime} \approx 0.1 \mathrm{~cm}^{-1}$. Since $\gamma^{\prime} \mathrm{x}$ is small, $e^{\gamma^{\prime} x} \approx 1$, and an exact knowledge of $\gamma^{\prime}$ is not required. We derive $\gamma^{\prime \prime}=0.36 \mathrm{~cm}^{-1}$. Inserting this value in Eq. (4) we obtain a detection limit of $6.6 \times 10^{-7} \mathrm{~nm} \cdot \sqrt{\mathrm{W} / \mathrm{Hz}}$. Both approaches give the same result, showing that our signal to noise ratio is indeed limited by photon noise.

$$
\text { We finally obtain } \quad \gamma^{\prime \prime}=0.39 \pm 0.03 \mathrm{~cm}^{-1} \quad \text { and } \quad \delta_{\lim }=(6.2 \pm 0.4) \times 10^{-}
$$

${ }^{7} \mathrm{~nm} \cdot \sqrt{\mathrm{W} / \mathrm{Hz}}$ (experimental point in Fig. 1). 
From the electron-hole competition model [23] and the B23 crystal parameters given in Table 1, we can compute $\gamma_{\text {th }}{ }^{\prime}=0.72 \mathrm{~cm}^{-1}$ and $\gamma_{\text {th }}{ }^{\prime \prime}=0.97 \mathrm{~cm}^{-1}$ for an applied electric field $\mathrm{E}_{0}=8 \mathrm{kV} \cdot \mathrm{cm}^{-1}$ and a grating spacing of $\Lambda=3.5 \mu \mathrm{m}$. Crystal parameters such as effective trap concentration $\left(\mathrm{N}_{\mathrm{eff}}\right)$ and electronhole competition factor $\left(\xi_{0}\right)$ were determined by studying the two wave mixing gain as a function of grating spacing (no applied field). $\xi_{0}$ is positive when the electrons are the majority charge carriers. Using $\alpha / \gamma^{\prime \prime}=1.9\left(\alpha=1.85 \mathrm{~cm}^{-1}\right)$, one predicts a detection limit $\delta_{\lim }=2.4 \times 10^{-7} \mathrm{~nm} \cdot \sqrt{\mathrm{W} / \mathrm{Hz}}$. This is almost 3 times better than our experimental value. It is to be expected that the electric field in the center of the crystal does not correspond to $\mathrm{V} / \mathrm{d}=8 \mathrm{kV} \cdot \mathrm{cm}^{-1}$, as the electric field can be reduced by screening effects caused by trapped space-charge [24]. A parallel electro-optic measurement of the Pockels effect, i.e a determination of the crystal transmission when placed between crossed polarizers and in the same experimental conditions (illumination and applied voltage) showed that the screening effects lead to a decrease of the internal field in the middle of the crystal by a factor of about 0.7 . Thus the real applied DC electric field is approximately $5 \mathrm{kV} \cdot \mathrm{cm}^{-1}$ which gives $\gamma_{\text {th }}{ }^{\prime}=0.45 \mathrm{~cm}^{-1}$ and $\gamma_{\text {th }}{ }^{\prime \prime}=0.80 \mathrm{~cm}^{-}$ ${ }^{1}$. Screening effects are not the only explanation for the smaller experimental gain value. This gain reduction could come from crystal imperfections like polycrystallinity or the existence of multiple trapping centers.

With the same experimental process in the B25 sample, we obtain a detection limit of $(8.2 \pm 0.5) \times 10^{-7} \mathrm{~nm} \cdot \sqrt{\mathrm{W} / \mathrm{Hz}}$ which corresponds to $\gamma^{\prime \prime}=0.22 \mathrm{~cm}^{-1}$. We note that with this sample we can not apply an electric field higher than $5 \mathrm{kV} \cdot \mathrm{cm}^{-1}$ to prevent electrical breakdown. Using the parameters of Table 1 for B25 and ignoring electric field screening effects leads to $\gamma_{\text {th }}{ }^{\prime \prime}=1.06 \mathrm{~cm}^{-1}$. Similarly to what we observed in B23, the measurement gives a reduced value of the gain.

To measure the adaptability of our system to deformations of the wavefront of the signal beam, we placed the diffuser in the signal path (Fig. 2). The étendue of the signal beam is defined as $G=\pi S \sin ^{2} \rho$, where $S$ and $\rho$ are the surface of the image and the half angle of the conical signal beam in front of the crystal, respectively. The étendue of a diffraction limited gaussian beam is given by $G=\lambda^{2}$ and is equal to $2.4 \times 10^{-6} \mathrm{~mm}^{2} \cdot \mathrm{sr}$. In our experiment with the diffuser in place, $\mathrm{G}$ was limited by the diameters of the lenses (f-number $=4$ ) and was equal to $\mathrm{G}=0.1 \mathrm{~mm}^{2} \cdot \mathrm{sr}$. This corresponds to an étendue $4 \times 10^{4}$ times higher than a gaussian beam. This value of $0.1 \mathrm{~mm}^{2} \cdot \mathrm{sr}$ is large enough for the multimode optical fibers 
generally employed in this kind of system. Within the measurement accuracy of $5 \%$, the signal beam intensity modulation observed after the crystal for a given phase modulation was the same for a gaussian signal beam and for the speckled beam produced by the diffuser in the signal beam path.

We have also experimentally studied the frequency response of the system, including at modulation periods larger than the photorefractive response time. The experiment was performed without the diffuser in the signal beam path. The results for both crystal samples are shown in Fig. 3. Using a $66 \mathrm{~mW} \cdot \mathrm{cm}^{-2}$ illumination intensity we obtained a cut-off frequency between 3 and $4 \mathrm{~Hz}$ for B23 and of $15 \mathrm{~Hz}$ for the B25. A slight overshoot was observed at about $15 \mathrm{~Hz}$ and $200 \mathrm{~Hz}$ for the B23 and B25 samples, respectively (Fig. 3). Theoretical description will be given in the following discussion.

\section{Discussion.}

Under CW illumination, the photorefractive response time is expected to be linear with the illumination intensity, the cut-off frequency should be around $1 \mathrm{kHz}$ at an illumination intensity of $19 \mathrm{~W} \cdot \mathrm{cm}^{-2}$ or $4.4 \mathrm{~W} \cdot \mathrm{cm}^{-2}$ for B23 and B25, respectively. Our samples have a much too high absorption to be used at these power densities. However, as shown in Ref. [16], this absorption contains a large residual part which can be eliminated with a new process of crystal growth.

It is clear from the curves in Fig. 1 that the best results will be obtained when the crystal absorption is very small. However, as the photorefractive effect relies on charge generation from light absorption, absorption can not be reduced too much. It is thus important to consider its influence on the detected signal when increasing the crystal length. As shown by Fig. 1, there is an optimal crystal thickness $\mathrm{x}_{\mathrm{opt}}$ for which the signal to noise ratio is minimal. From Eq. (4), we obtain $x_{o p t}=\frac{1}{\gamma^{\prime \prime}} \operatorname{Arctan} \frac{2 \gamma^{\prime \prime}}{\alpha}$. When the crystal thickness exceeds this limit, the signal reduction due to absorption becomes more important than the signal increase due to amplification by the diffracted part of the pump beam. The detection limit also depends on crystal geometry. Its minimal value, and the maximum value of $\gamma^{\prime \prime}$, is obtained when the crystal orientation is such that $r_{\text {eff }}=2 r_{41} / \sqrt{3}$.

If the B23 crystal sample had such an optimized orientation, we would obtain $\gamma^{\prime \prime}=0.54 \mathrm{~cm}^{-1}$. Given the crystal absorption $\alpha=1.85 \mathrm{~cm}^{-1}$, the minimum of $\delta_{\text {lim }}$ in Eq. (4) is reached for $x_{o p t}=1 \mathrm{~cm}$. One thus expects a detection limit $\delta_{\lim }=1.8 \times 10^{-7} \mathrm{~nm} \cdot \sqrt{\mathrm{W} / \mathrm{Hz}}$ that is more than three times better than our 
current experimental value. The parameter $\alpha / \gamma^{\prime \prime}$ of a crystal is the only constraint on the detection limit. The large background absorption of B23 makes $\alpha / \gamma^{\prime \prime}$ equal to 3.4, which is too high for going beyond the threshold of $10^{-7} \mathrm{~nm} \cdot \sqrt{\mathrm{W} / \mathrm{Hz}}$. This is the second motivation for growing crystals with less absorption.

The crystal absorption has also an effect on the frequency response of the crystal. This can be calculated by considering the full expression for the response of the system, valid whatever the relative amplitude between the period of the modulation and the photorefractive response time. Using the same nomenclature as in Eqs. (1-2), the amplitude of the signal beam after the crystal is given by [13] :

$$
\begin{aligned}
& \left.\mathrm{E}_{\mathrm{s}}(\mathrm{x}, \mathrm{t})=\mathrm{e}^{-\frac{\alpha \mathrm{x}}{2}} \mathrm{e}^{\gamma \mathrm{x}} \mid \mathrm{E}_{\mathrm{s}}(0,0)+\int_{0}^{\mathrm{t}}\left(\tau_{0} \frac{\partial \mathrm{E}_{\mathrm{s}}}{\partial \mathrm{t}}(0, \mathrm{~T})+\mathrm{E}_{\mathrm{s}}(0, \mathrm{~T})-\mathrm{E}_{\mathrm{s}}(0,0)\right) \mathrm{H}(\mathrm{x}, \mathrm{t}-\mathrm{T}) \mathrm{dT}\right], \\
& \text { and } \quad H(x, t)=\frac{e^{-\gamma x}}{\tau_{0}} e^{-\frac{t}{\tau_{0}}}{ }_{1} \mathrm{~F}_{1}\left(\frac{\gamma}{\alpha}, 1,\left(\frac{e^{\alpha x}-1}{e^{\alpha x}}\right) \frac{t}{\tau_{0}}\right),
\end{aligned}
$$

where $\tau_{\mathrm{o}}$ is the photorefractive time constant at the entrance of the crystal $(\mathrm{x}=0)$, and ${ }_{1} \mathrm{~F}_{1}(\mathrm{a}, \mathrm{b}, \mathrm{z})$ is the confluent hypergeometric function. The signal beam is phase modulated at a frequency $\mathrm{f}$ : $\varphi(t)=\varphi_{0} \cos (2 \pi f \mathrm{t})$ with $\varphi_{0}<<\pi / 2$. From Eqs. (5-6), we compute the intensity modulation of the signal beam as a function of the frequency $\mathrm{f}$. The theoretical response is calculated for B23 and B25 and for a $66 \mathrm{~mW} \cdot \mathrm{cm}^{-2}$ illumination using the parameters of Table 1 . The modulus and phase of the response time of the crystal under DC field are experimentally determined by measuring the kinetics of energy transfer when the DC field amplitude is reversed with a periodicity slower than the response time of the crystal. Adjustment of experimental results with theoretical expressions (Eq. (5) in Ref. [25]) allows to determine both modulus and phase of the crystal response time $\tau$ (Table 1 ). Since $\alpha x<1, \tau_{0}$ is obtained from $\tau_{0}=\tau \cdot e^{-\alpha x / 2}[13]$.

For both samples, the theoretical curves are drawn using the measured gain and time constant, without any adjustments to the frequency response data. The photorefractive beam-combiner acts as a high pass filter with an overshoot. An increase of absorption produces a decrease of the cut-off frequency and a reduction of the height of the overshoot. This theoretically expected slight overshoot in the frequency response was also observed experimentally. For B23, we present two other theoretical curves where we use a phase of the response time of $0.4 \mathrm{rad}$ and $1 \mathrm{rad}$ instead of $0.7 \mathrm{rad}$. These two values correspond to our measurement uncertainty. We see that a small variation of the phase of the response 
time produces a large variation of the height of the overshoot. It allows us to tell that frequency response measurements give a more accurate determination of the phase of the response time which was otherwise quite inaccurate. An examination of Eq. (5) shows that a change in the modulus of the response time leads to a lateral shift of the whole curve without modifying its shape.

\section{Conclusion}

We characterized a homodyne detection system using a CdTe crystal as the element which combines the local oscillator beam with the signal beam. We obtained a detection limit approaching the theoretical limit and demonstrated the insensitivity of the system to deformations in the signal beam wavefront up to an étendue of $0.1 \mathrm{~mm}^{2} \cdot \mathrm{sr}$. We studied the response of the system as a function of the phase modulation frequency in the signal beam and demonstrated the expected high band pass response.

We now plan to test the system for a signal beam with a larger étendue of the order of some $\mathrm{mm}^{2} \cdot \mathrm{sr}$. We will also approach the ideal interferometric detection limit (with $\delta_{\lim } \leq 10^{-7} \mathrm{~nm} \cdot \sqrt{\mathrm{W} / \mathrm{Hz}}$ ) by using less absorbing crystals $\left(\alpha / \gamma^{\prime \prime} \leq 1\right)$ (Fig. 1). In such low-absorbing crystals we also expect to obtain a cut-off detection frequency of $1 \mathrm{kHz}$ at illumination densities of the order of some $\mathrm{W} \cdot \mathrm{cm}^{-2}$.

\section{References}

[1] T.J. Hall, M.A. Fiddy, M.S. Ner, Opt. Lett. 5 (1980) 485.

[2] A.E. Siegman, Appl. Opt. 5 (1966) 1588.

[3] J.-P. Monchalin, R. Héon, P. Bouchard and C. Padioleau, Appl. Phys. Lett. 55 (1989) 1612.

[4] F. Davidson and L. Boutsikaris, Opt. Eng. 29 (1990) 369.

[5] G. Hamel de Monchenault and J.-P. Huignard, J. Appl. Phys. 63 (1988) 624.

[6] Y.O. Barmenkov, V.V. Zosimov, N.M. Kozhevnikov, L.M. Lyamshev and S.A. Sergushchenko, Sov. Phys.-Dokl. 31 (1986) 817.

[7] S. Stepanov and I. Sokolov in Proc. of II Intern. Conf. on Holographic Systems, Components and Applications (Bath, UK, 1989) 95.

[8] R.K. Ing and J.-P. Monchalin, Appl. Phys. Lett. 59 (1991) 3233.

[9] C.T. Field and F.M. Davidson, Appl. Opt. 32 (1993) 5285. 
A. Blouin and J.-P. Monchalin, Appl. Phys. Lett. 65 (1994) 932.

[11] A.A. Kamshilin, J. Frejlich and L. Cescato Appl. Opt. 25 (1986) 2375.

[12] L.-A. de Montmorillon, Ph. Delaye, I. Biaggio, J.-C. Launay and G. Roosen, in Digest of Topical Meeting on Photorefractive Materials, Effects and Devices, Aspen Lodge PR95 (1995) 504.

[13] Ph. Delaye, L.-A. de Montmorillon and G. Roosen, Opt. Commun. 118 (1995) 154.

[14] D.B. Chenault, R.A. Chipman and S.Y. Lu, Appl. Opt. 33 (1994) 7382.

[15] A. Partovi, J. Millerd, E.M. Garmire, M. Ziari, W.H. Steier, S.B. Trivedi and M.B. Klein, Appl. Phys. Lett. 57 (1990) 846.

[16] L.-A. de Montmorillon, Ph. Delaye, J.-C. Launay and G. Roosen, Opt. Mat. 4 (1995) 233.

[17] J.F. Nye, Physical Properties of Crystals (Clarendon, Oxford, 1957) chapter 13.

[18] A.J. Strauss, Rev. Phys. Appl. 12 (1977) 167.

[19] P. Maheswarananthan, R.J. Sladek and U. Debska, Phys. Rev. B 31 (1985) 7910.

[20] S. Stepanov, S.Shandarov and N. Khat'kov, Sov. Phys. Solid State 29 (1987) 1754.

[21] G. Pauliat, P. Mathey and G. Roosen, J. Opt. Soc. Am. B 8 (1991) 1942.

[22] P. Günter and M. Zgonik, Opt. Lett. 16 (1991) 1826.

[23] ～F.P. Strohkendl, J.-M. C.Jonathan, R.W. Hellwarth, Opt. Lett. 11 (1986) 312.

[24] J.-M. C.Jonathan, R.W. Hellwarth and G. Roosen, IEEE J. of Quantum El. 22 (1986) 1936.

[25] C. Besson, J.-M. C.Jonathan, A. Villing, G. Pauliat and G. Roosen, Opt. Lett. 14 (1989) 1359.

[26] J.-P. Monchalin and R. Héon, Mater. Eval. 44 (1986) 1231. 


\section{Table Caption}

Table 1: Measured characteristics of the photorefractive crystals. The response time and the photorefractive gain were measured with a DC applied electric field of $8 \mathrm{kV} \cdot \mathrm{cm}^{-1}$ for B23 and $5 \mathrm{kV} \cdot \mathrm{cm}^{-1}$ for $\mathrm{B} 25$. The absorption at $\lambda=2 \mu \mathrm{m}$ corresponds to a residual background absorption [16]. 


\section{Figure Caption}

Fig. 1: Theoretical detection limit as a function of the $\gamma^{\prime \prime} \mathrm{x}$ factor. The left vertical scale gives the detection limit resulting from Eq. (4) using a quantum efficiency of 0.76. The right vertical scale gives the relative detection limit that is normalized to the plane wave interferometric detection limit. The different curves correspond to different $\alpha / \gamma^{\prime \prime}$ values. The dashed line corresponds to the detection limit of the confocal Fabry-Perot used in transmission [26]. The circle corresponds to the experimental measurement with B23. The square corresponds to the typical sensitivity we expect to obtain with an optimized crystal.

Fig. 2: Experimental set-up used for the study of the homodyne detection system.

Fig. 3: Normalized signal, i.e. amplitude of the intensity modulation normalized to the high frequency value, versus frequency. The empty circles and full triangles give the experimental results in samples B23 and B25, respectively. The thick lines give for both crystals the prediction obtained from Eq. (5) and the parameters in Table 1. The dashed line and the dashdotted line give the result for the B23 parameters in Table 1, but with $0.4 \mathrm{rad}$ and $1 \mathrm{rad}$ for the phase of the response time, respectively.

The insert shows the detected signal amplitude as a function of the DC applied electric field. 


\begin{tabular}{|c|c|c|}
\hline & $\mathrm{B} 23$ & $\mathrm{~B} 25$ \\
\hline$\alpha(1.55 \mu \mathrm{m})$ in $\mathrm{cm}^{-1}$ & 1.85 & 2.31 \\
\hline$\alpha(2 \mu \mathrm{m})$ in $\mathrm{cm}^{-1}$ & 1.27 & 0.8 \\
\hline$\xi_{0}(1.55 \mu \mathrm{m})$ & $-0.83 \pm 0.05$ & $+0.63 \pm 0.03$ \\
\hline $\mathrm{N}_{\mathrm{eff}}(1.55 \mu \mathrm{m})$ in $\mathrm{cm}^{-3}$ & $(1.5 \pm 0.2) \times 10^{15}$ & $(5.0 \pm 0.4) \times 10^{15}$ \\
\hline $\mathrm{r}_{\mathrm{eff}}$ in $\mathrm{pm} \cdot \mathrm{V}^{-1}$ & 4.5 & 5.5 \\
\hline crystal thickness in $\mathrm{cm}$ & 0.18 & 0.27 \\
\hline Response time in ms & $(13 \pm 5) \times \mathrm{e}^{\mathrm{i}(0.7 \pm 0.3)}$ & $(4 \pm 2) \times \mathrm{e}^{\mathrm{i}(0.7 \pm 0.3)}$ \\
\hline$\gamma$ in $\mathrm{cm}^{-1}$ & $(0.4 \pm 0.02) \times \mathrm{e}^{\mathrm{i}(1.4 \pm 0.1)}$ & $(0.3 \pm 0.02) \times \mathrm{e}^{\mathrm{i}(1.4 \pm 0.1)}$ \\
\hline
\end{tabular}

Table 1 - Louis-Anne de Montmorillon 


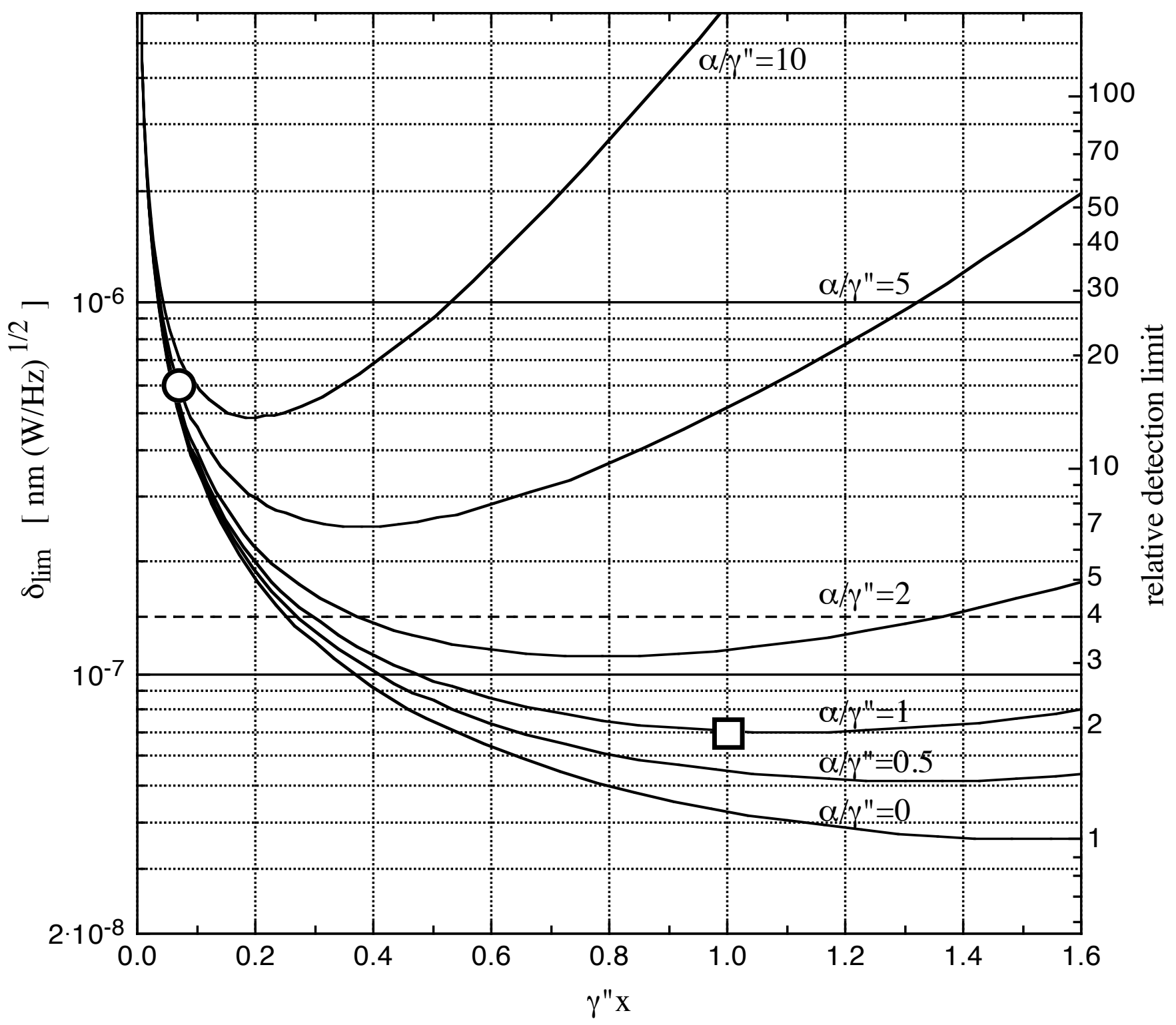

Fig. 1 - Louis-Anne de Montmorillon 


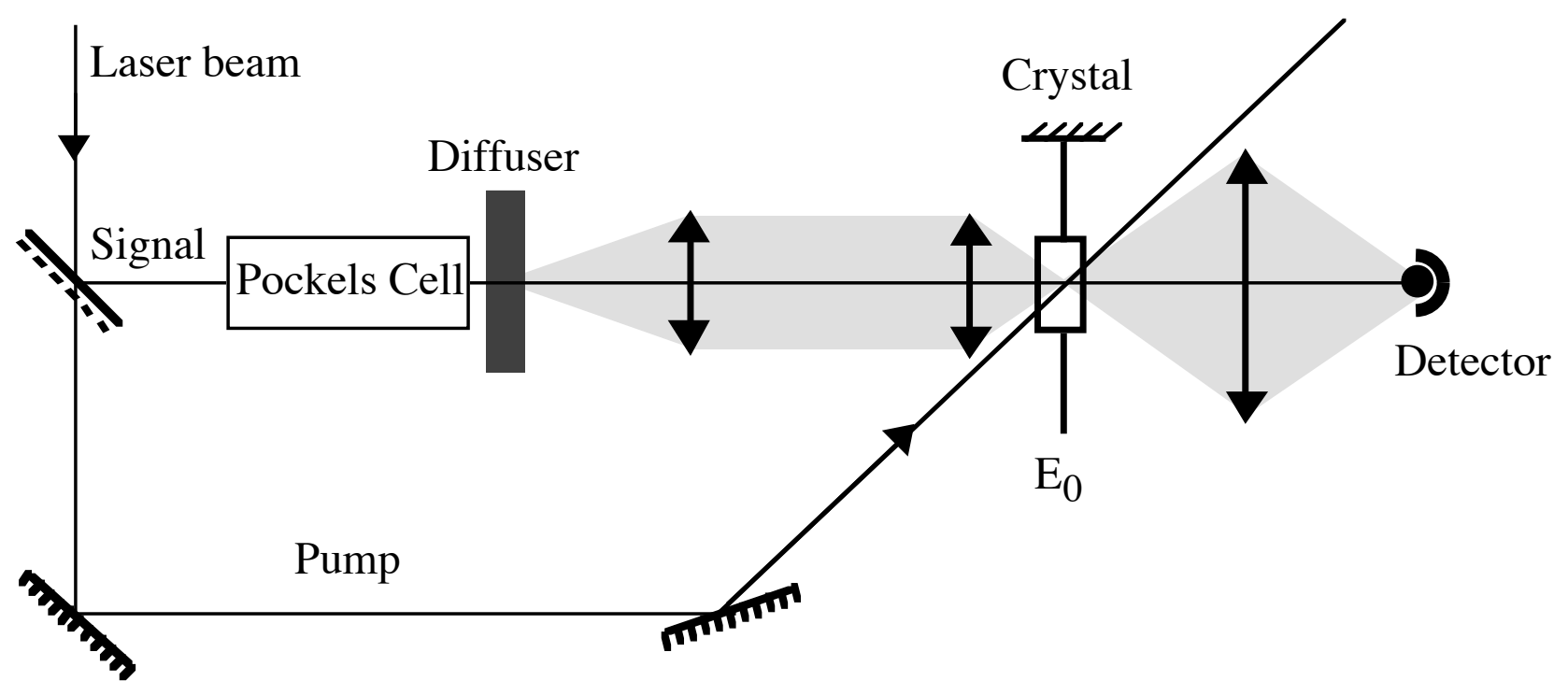

Fig. 2 - Louis-Anne de Montmorillon 


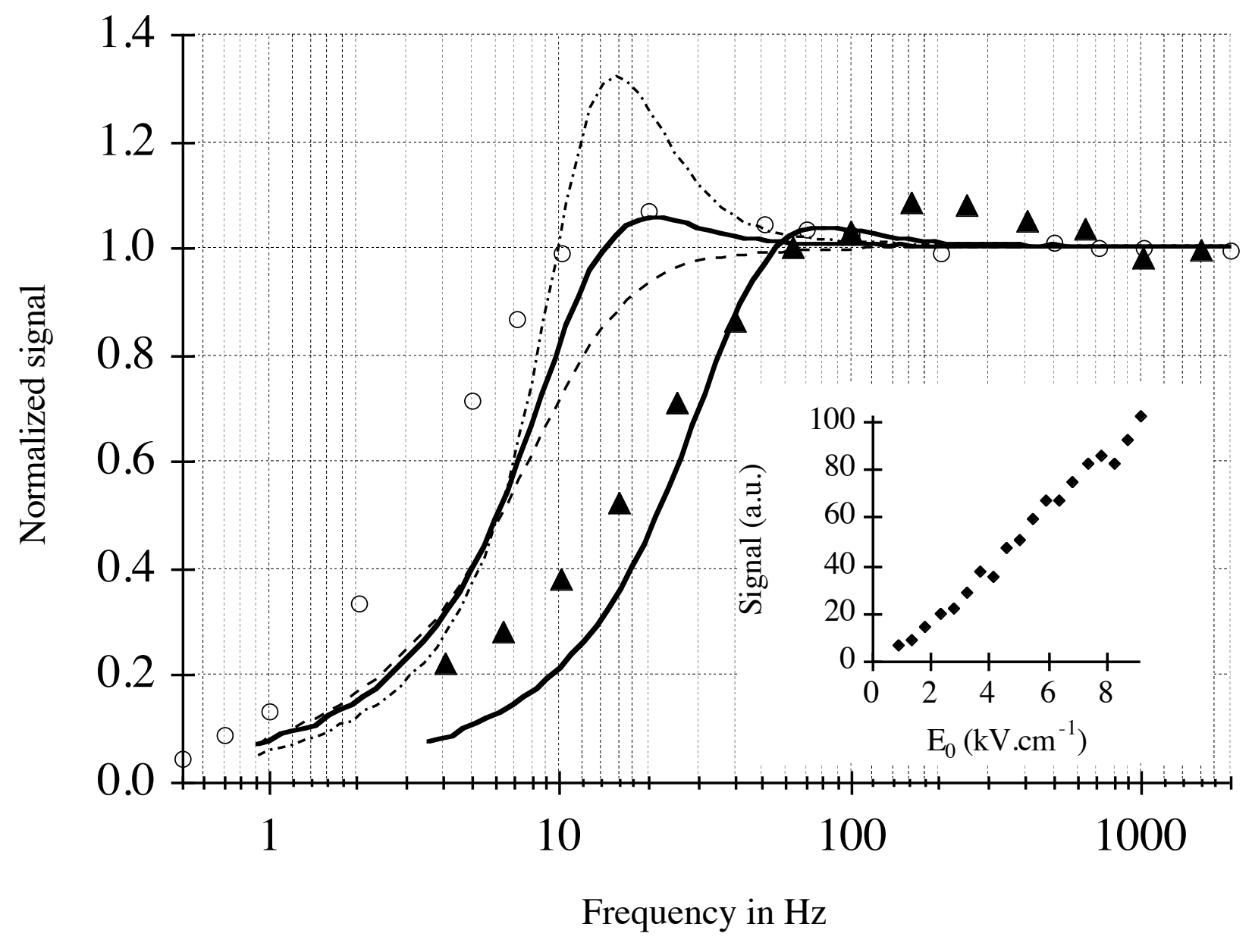

Fig. 3 - Louis-Anne de Montmorillon 